\title{
A QTL with Major Effect on Reducing Stripe Rust Severity Detected From a Chinese Wheat Landrace
}

H. Y. Zhang, Z. Wang, and J. D. Ren, Department of Plant Pathology, China Agricultural University, Beijing 100193, P. R. China; Z. Y. Du, The Open University of China, Beijing 100039, P. R. China; W. Quan, Beijing Engineering and Technique Research Center for Hybrid Wheat, Beijing Academy of Agricultural and Forestry Sciences, Beijing 100097, P. R. China; and Y. B. Zhang and Z. J. Zhang, Department of Plant Pathology, China Agricultural University, Beijing 100193, P. R. China

\begin{abstract}
Stripe rust, a devastating disease of wheat worldwide, can be controlled by use of diverse wheat resistance resources. To find new quantitative trait loci (QTL) for resistance to stripe rust, Qing Shumai (a Chinese winter wheat landrace possessing slow rusting resistance) was crossed with the susceptible line Mingxian 169. The parents and 276 recombinant inbred lines (RILs) from the cross were evaluated in five environments involving two locations (Gansu and Shandong provinces, China) and four autumn-sown wheat seasons (2008 to 2012). Disease severities on Qing Shumai were lower than $25 \%$, contrasting with approximately $90 \%$ on Mingxian 169. The RILs varied in rust intensity in a continuous and

monomodal distribution. A bulked segregant analysis approach using 2,344 simple sequence repeat (SSR) markers mapped a major QTL to the long arm of chromosome 6D (hereby designated as QYr.cau- $6 D L$ ). An SSR marker (gpw5179, https://wheat.pw.usda.gov/GG2/index.shtml) was identified as being tightly linked with $Q Y$ r.cau-6DL. Combination between $Q Y r$.cau- $6 D L$ and the stripe rust-resistance gene $Y r 18$ was examined using $160 \mathrm{~F}_{2: 3}$ families of Qing Shumai $\times$ RL6058 (a near-isogenic line for Yr18 in the genetic background of the spring wheat Thatcher). The combination elevated the resistance consistently across both winter and spring wheat backgrounds, acting synergistically without undesired epistasis.
\end{abstract}

Stripe or yellow rust, caused by the fungus Puccinia striiformis Westend. f. sp. tritici Erikss., is one of the most destructive diseases of common wheat (Triticum aestivum L.) worldwide (Hovmøller et al. 2008; Wellings 2011). Utilization of wheat resistance resources can reduce the need for fungicides, and thus is an economical and environmentally sound strategy for controlling this disease (Johnson 1992). A great effort has been dedicated to searching for stripe rust-resistance resources globally, and 74 officially designated stripe rust-resistance $(Y r)$ genes have been cataloged in addition to more than 40 temporary designations (Dracatos et al. 2016; McIntosh et al. 2013a, b). Of these $\mathrm{Yr}$ genes, most act in a gene-for-gene nature as observed in many other plant-pathogen systems (Dangl et al. 2013; Flor 1971), and they are vulnerable to adaptation by new virulent races of $P$. striiformis. Many race-specific $Y r$ genes such as $Y r 2$, $Y r 9$, and $Y r 27$, after being used in commercial wheat fields for a short period of time, have become ineffective in combating the current P. striiformis populations (Wellings 2011).

In contrast, $\operatorname{Yr} 18$ has remained durable, and no evolution of increased virulence toward it has been observed for more than 50 years (Dyck et al. 1966; Krattinger et al. 2009; McIntosh 1992). Unlike race-specific $\mathrm{Yr}$ genes that typically condition a degree of hypersensitive response leading to low infection types either in the seedling or the adult-plant growth stages, $\operatorname{Yr} 18$ represents another type that exhibits high infection types but confers resistance in a quantitative manner (partial resistance) predominantly manifested in the post-seedling stage (Johnson 1992; Singh 1992). This type of resistance is often quantified by lower disease severity or smaller area under the disease progress curve (AUDPC) compared with a susceptible check genotype (Milus and Line 1986; Singh 1992). This resistance can be genetically mapped in chromosome regions delimited by DNA markers

Corresponding author: Z. J. Zhang; E-mail: zhangzj@cau.edu.cn

Z. Wang and J. D. Ren contributed equally to this study.

*The $\boldsymbol{e}$-Xtra logo stands for "electronic extra" and indicates that one supplementary table and two supplementary figures are published online.

Accepted for publication 7 April 2017.

C) 2017 The American Phytopathological Society through quantitative trait loci (QTL) mapping approaches, and it can be selected on the basis of tightly linked DNA markers. Rosewarne et al. (2013) provided a review about QTL for stripe rust-resistance in wheat, summarizing more than 140 QTL, and additional QTL were reported more recently (e.g., Maccaferri et al. 2015). Diverse QTL illustrate substantial genetic diversity for this trait, although some of them may be redundant. Some QTL display larger effect (e.g., explaining $>20 \%$ of phenotypic variance individually) and higher stability in effectiveness across diverse genetic backgrounds and environments than others. The term "major QTL" is often used to indicate a QTL that has a larger effect. QTL have been found to confer durable resistance in several cases such as $\operatorname{Yr} 18$ and $Y r 29$, even though exceptions were reported (Singh et al. 2011). From the viewpoint of practical breeding, a major QTL with high stability and durability could be more valuable than others. Of these QTL, Yr18 is the most intensively studied, and a series of gene-specific DNA markers (e.g., cssfr5) have been developed from the cloned $\operatorname{Yr} 18$ sequences, which can be applied to selecting for Yr18 unambiguously (Krattinger et al. 2009; Lagudah et al. 2009). Combination of different QTL can yield a high level resistance (Singh et al. 2000), and DNA marker selection provides a powerful tool for combining QTL.

It has been suggested that over-reliance on a limited number of resistance genes/QTL would be imprudent for deployment over vast wheat-growing regions (McDonald and Linde 2002; William et al. 2006). Therefore, it is essential to detect additional stripe rustresistance QTL to increase the diversity of resistance resources for sustainably controlling this disease. We previously reported that the Chinese wheat landrace Qing Shumai possesses slow stripe rusting resistance at the adult plant growth stage (Z. J. Zhang 1995). The objectives of this study were to (i) detect QTL associated with the stripe rust-resistance of Qing Shumai, (ii) screen for a DNA marker tightly linked to $Q Y$ r.cau- $6 D L$ and examine the applicability of the marker gpw5179 for selection for QYr.cau-6DL, and (iii) examine the effect of the combination between $Q Y$ r.cau- $6 D L$ and Yr18 in both spring wheat and winter wheat genetic backgrounds.

\section{Materials and Methods}

Wheat population for mapping QTL and assessment of stripe rust-resistance. The wheat mapping population consisted of 276 recombinant inbred lines (RILs) derived from the cross Mingxian $169 \times$ Qing Shumai. Both parents are landraces with no pedigree data 
being available. Qing Shumai exhibited consistently low stripe rust severities (7 to $30 \%$ ) at the adult plant growth stage in our field nurseries located in a "hotspot" area of P. striiformis (Z. J. Zhang 1995). Mingxian 169 is highly susceptible to all of the $P$. striiformis races detected in China (W. Q. Chen et al. 2009; Wan et al. 2004). The construction of the mapping population began in May 1996. The 276 RILs derived from a single $F_{1}$ seed were advanced to $F_{6}$ plants by single-seed descent. From the $\mathrm{F}_{6}$ plants of each RIL, one spike was sampled to grow $\mathrm{F}_{7}$ spike-row plants. An individual seed was then sampled from the spike-row to serve as the representative of $F_{8}$ generation, and the remaining seeds of the spike-row were bulked to grow $\mathrm{F}_{6: 8}$ plants. Likewise, the representative seed and bulked seeds of the subsequent generations were developed. Mingxian 169 was used as the susceptible control throughout this study.

The 276 RILs were tested for stripe rust-resistance in commercial fields in southern Gansu Province (Wushan County; 34 $42^{\prime} 15^{\prime \prime} \mathrm{N}$, $104^{\circ} 40^{\prime} 08^{\prime \prime} \mathrm{E}$, elevation $1,680 \mathrm{~m}$ ) in four autumn-sown wheat seasons (2008-09 to 2011-12) and in Shandong (Tai'an; 36 $18^{\prime} 09^{\prime \prime}$ $\mathrm{N}, 117^{\circ} 13^{\prime} 05^{\prime \prime}$ E, elevation $90 \mathrm{~m}$ ) in $2011-12 . \mathrm{F}_{6: 8}, \mathrm{~F}_{7: 9}, \mathrm{~F}_{8: 10}$, and $\mathrm{F}_{9: 11}$ generations were used in 2008-09, 2009-10, 2010-11, and 2011-12 seasons, respectively. Sowing was done in mid-September in southern Gansu and in early October in Shandong. In each of the five test environments, the trial was in a randomized complete block design with three replicates. An individual plot consisted of a single $1 \mathrm{~m}$ long row with $25 \mathrm{~cm}$ between adjacent rows. Each plot was sown with approximately 40 seeds of an RIL. A set of parents were included after every 60 rows for checking homogeneity of $P$. striiformis infection. In Shandong, a row of Mingxian 169 perpendicular and adjacent to the plot rows was sown along each field block to serve as spreader plants.

The environments of southern Gansu allow P. striiformis to survive year-round and are highly conducive for stripe rust epidemics. Winter wheat is sown in mid-September and harvested late in the following July. Volunteer wheat seedlings provide a "green bridge" for $P$. striiformis to move from the harvested wheat plants to the autumnsown seedlings. To reinforce the green bridge, the areas bordering the trial plots were sown with Mingxian 169 in early July. The autumnsown test seedlings were infected with the urediniospores (i.e., the naturally occurring $P$. striiformis populations) released from the green bridge plants. The infected seedlings were covered with plastic film during winter months for facilitating $P$. striiformis to overwinter and thus to increase infection in the forthcoming spring. In the cases of drought in spring and/or summer, the test plants were frequently misted after sunset to help reinfection as well as provide regular irrigation.

In Shandong, the hot summer is lethal to $P$. striiformis, and artificial inoculation is necessary. The Chinese $P$. striiformis race CYR32 was used as the inoculum, which is currently the most predominant race in China with the avirulence/virulence formula $\operatorname{Yr} 5, \operatorname{Yr} 8$, Yr10, Yr15, Yr26/Yr1, Yr2, Yr3, Yr4, Yr6, Yr7, Yr9, Yr22, Yr23, Yr25, Yr27, YrA, YrAlba, YrCle, YrCV1, YrCV2, YrCV3, YrGaby, YrRes, $\mathrm{YrSD}, \mathrm{YrSO}, \mathrm{YrSpP}$, and $\mathrm{YrSu}$ (Li et al. 2006; Wan et al. 2004). An initial inoculum of CYR32 was kindly provided by Professors W. Q. Chen and S. C. Xu at the Institute of Plant Protection, Chinese Academy of Agricultural Science, Beijing. Urediniospores were propagated on Mingxian 169 plants. Inoculation was done in midMarch when the test plants were at the stem elongation stage. On a clear afternoon when there was a high likelihood of overnight dew, the spreader plant shoots were injected using syringes with fresh urediniospores suspended in water containing $0.04 \%$ Tween 20 (approximately 80,000 uridiniospores/ml, $100 \mu \mathrm{l} /$ injection). Twenty days after inoculation when some urediniospores were obviously released from the spreader plants, the test plants were frequently misted after sunset.

Stripe rust severity in the form of percentage of infected leaf area was scored on flag leaves using the modified Cobb scale (Peterson et al. 1948). The estimation was on the plot basis, viz., by visually averaging over all the flag leaves of each plot entry. Recording was done three times when the severities on Mingxian 169 flag leaves reached approximately 10 to $30 \%, 50$ to $60 \%$, and 80 to $90 \%$, respectively. AUDPC was calculated using the three recordings for each plot entry as described in Du et al. (2015). QTL mapping was based on mean AUDPC calculated for each RIL by averaging over all replicates within individual environments.

Additionally, Qing Shumai was tested for consistency of resistance during a period of 22 years (from 1995 to 2016) in southern Gansu. In each year, Qing Shmai was planted in three replicates in field plots with Mingxian 169 as a susceptible control, infected with the naturally occurring $P$. striiformis populations. An individual plot consisted of a $1 \mathrm{~m}$ long row. From the central part of each plot, five flag leaves were scored for disease severity using the modified Cobb scale (Peterson et al. 1948). Disease mean was calculated over the 15 leaves of three replicates within a year for each wheat line.

Construction of genetic linkage map and QTL analysis. The map construction and QTL analysis were performed using the 276 RILs by the procedures as described in Du et al. (2015). Briefly, DNA was extracted using the CTAB method. SSR and EST (expressed sequence tag) primer sequences were acquired from the public domains (e.g., https://wheat.pw.usda.gov/GG2/index.shtml). A bulked segregant analysis approach was applied to the population. Twenty RILs were selected which showed the lowest stripe rust severities consistently in the three test seasons of 2008-09 $\left(\mathrm{F}_{6: 8}\right)$, 2009-10 $\left(\mathrm{F}_{7: 9}\right)$, and 2010-11 $\left(\mathrm{F}_{8: 10}\right)$, and 20 RILs showing the highest severities were selected. DNA samples from $F_{10}$ plants of the selected RILs were used to construct the resistant (R-) and susceptible (S-) bulks. In total, 2018 SSR and EST primer pairs were screened against the R- and S-bulks for clearly distinct and readily repeatable polymorphic bands that were then used to genotype the $276 \mathrm{~F}_{10}$ RILs. Linkage analysis was carried out using the software MAPMAKER/ EXP 3.0 (Lander et al. 1987) set to Kosambi mapping function in centiMorgans (cM). QTL mapping was done using the composite interval mapping (CIM) method in Windows QTL Cartographer 2.5 (Wang et al. 2010). This software was also used to calculate the coefficient of determination $\left(R^{2}\right)$ and the threshold logarithm of the odds (LOD) score for declaring significant QTL. $R^{2}$ is a measurement of effect size, viz., proportion of phenotypic variance explained by a QTL. The threshold LOD score was calculated by running the permutation program set with 3,000 replications at a type I error rate of $\alpha=0.01$. Permutation analyses were conducted for each of the five test environments and, for simplicity, the highest threshold LOD value (4.2) was chosen as a uniform threshold for all environments.

Population for examining the combination between QYr.cau6DL and $\mathrm{Yr18}$. To examine the effect of the combination between QYr.cau-6DL and Yr18 (represented by the DNA markers gpw5179 and cssfr5, respectively) as well as the applicability of gpw5179 to select for QYr.cau-6DL, Qing Shumai was crossed with RL6058 (a backcross-derived near-isogenic line of the spring wheat cultivar Thatcher with Lr34; Dyck and Samborski 1982). The cross was done in May 2012. In mid-October 2013, 2,200 $\mathrm{F}_{2}$ seeds were sown in 5-ml centrifuge tubes filled with moist soil with a single germinated seed in each tube, maintained in growth chambers at $5 \pm 2{ }^{\circ} \mathrm{C}$ with a relative humidity higher than $70 \%$. Selection, based on gpw5179 for $Q Y$ r. cau-6DL and cssfr 5 for $Y r 18$, was done on the DNA samples extracted from the 2,200 seedlings. This selection resulted in four marker classes, namely, the $\mathrm{F}_{2}$ seedlings in homozygous state for the resistant alleles at both gpw5179 and cssfr5 (denoted with,++ ), resistant allele at gpw5179 but susceptible allele at cssfr5 (+, -), susceptible allele at gpw5179 but resistant allele at $\operatorname{css} f r 5(-,+)$, and susceptible alleles at both gpw5179 and cssfr5 (-, -).

One objective of this study was to examine these four marker classes in both spring and winter wheat genetic backgrounds. Preliminary experiments had indicated that the RL6058 × Qing Shumai population was segregating at the vernalization $(V r n)$ gene locus Vrn-Al while monomorphic at all other Vrn loci (Z. J. Zhang and associates, unpublished data). In this population, therefore, the spring growth habit is simply conferred by the allele Vrn-Ala (dominant), and the winter growth habit is determined by $v r n-A 1$ (recessive). Selection was done for $v r n-A 1$ and $V r n-A l a \mathrm{~F}_{2}$ plants using the same procedures as described in X. Zhang et al. (2008) based on the allele-specific markers developed from the cloned $V R N-1$ gene sequences (Fu et al. 2005; Yan et al. 2004) (Supplementary Table 
S1). From each of the four marker classes, 20 seedlings were selected which were in homozygous state for $v r n-A 1$, and another 20 seedlings in homozygous state for Vrn-Ala were selected. These 160 plants were then transplanted from the centrifuge tubes to the soil in a greenhouse in Inner Mongolia (Chifeng; 42 $16^{\prime} 36^{\prime \prime} \mathrm{N}, 119^{\circ} 01^{\prime}$ $37^{\prime \prime} \mathrm{E}$, elevation $560 \mathrm{~m}$ ) where greenhouse temperatures can be easily controlled to the degrees suitable for the tests. From late November 2013 to early February 2014, greenhouse heating was turned off, and on sunny days, the wheat seedlings were sheltered from sunlight. This resulted in the greenhouse temperatures of $-3^{\circ} \mathrm{C}$ (the lowest, night) to $7^{\circ} \mathrm{C}$ (the highest, day), a temperature range favorable for vernalizing wheat seedlings. Afterward, the greenhouse was conditioned at 10 to $21^{\circ} \mathrm{C}$ daily under natural daylight supplemented to $16 \mathrm{~h} . \mathrm{F}_{3}$ seeds were harvested separately from each of the $160 \mathrm{~F}_{2}$ plants in late May 2014.

The tests for resistance were conducted on the $80 \mathrm{~F}_{2: 3}$ families of spring growth habit in the 2014-15 wheat season and on the $80 \mathrm{~F}_{2: 3}$ families of winter growth habit in 2015-16. The trials were carried out in southern Gansu as described above with the four following exceptions. First, an individual plot consisted of 20 rows with one $\mathrm{F}_{2: 3}$ family in each row, sown with 20 seeds per family. Second, disease recording was done on eight flag leaves sampled from the central part of each row when Mingxian 169 was showing a disease severity of approximately $90 \%$. The mean severity of the eight leaves was considered as row value with the grand average over 20 rows as plot value. Third, a row of Mingxian 169 plants was included after every $10 \mathrm{~F}_{2: 3}$ family rows for checking infection homogeneity. Last, in the 2014-15 season when the $80 \mathrm{~F}_{2: 3}$ families of spring growth habit were tested, sowing was done in early March 2015.

Statistical analysis. All analyses were performed in SAS/STAT version 9.3 (SAS Institute Inc., Cary, NC). For the Mingxian 169× Qing Shumai population tested in the five field environments, an analysis of variance (ANOVA) was carried out based on AUDPC values using PROC GLIMMIX. A linear mixed model was fitted with all five environment trials combined: $P_{i j k}=\mu+G_{i}+E_{j}+E_{j}\left(R_{k}\right)+G_{i} \times E_{i}+$ $e_{i j k}$, where $P_{i j k}$ is the phenotypic value, $\mu$ the population mean, $G_{i}$ the effect of the $i$ th genotype (i.e., RIL), $E_{j}$ the effect of the $j$ th environment, $E_{j}\left(R_{k}\right)$ the effect of the $k$ th replicate within the $j$ th environment, $G_{i} \times E_{j}$ the $i j$ th effect of the genotype-by-environment interaction, and $e_{i j k}$ designated the residual. The effects of genotype, environment, and genotype-by-environment interaction were considered as fixed and the other terms as random effects. Broad-sense heritability $(H)$ was estimated using PROC GLIMMIX setting all effects as random and based on the equation $H=\sigma_{\mathrm{G}}^{2} /\left[\sigma_{\mathrm{G}}^{2}+\sigma_{\mathrm{G} \times \mathrm{E}}^{2} / \mathrm{m}+\sigma_{\text {error }}^{2} /(\mathrm{m} \times \mathrm{r})\right]$, where $\sigma_{\mathrm{G}}^{2}$ denotes the genotypic (i.e., RIL) variance, $\sigma_{\mathrm{G} \times \mathrm{E}}^{2}$ the genotype-by-environment interaction variance, $\sigma_{\text {error }}^{2}$ the error variance, $\mathrm{m}$ the environment number, and $\mathrm{r}$ the replicate number within each environment (Holland et al. 2003). To examine the variance within individual environments, an ANOVA was also performed separately for each of the five environment trials by fitting the model: $P_{i k}=\mu+G_{i}+R_{k}+e_{i k}$, where $P_{i k}$ is the phenotypic value, $\mu$ the population mean, $G_{i}$ the effect of the $i$ th genotype, $R_{k}$ the effect of the $k$ th replicate, and $e_{i k}$ the residual. The genotype and replicate effects were considered as fixed and the overall error term as random effect. $H$ was estimated based on the equation $H=\sigma_{\mathrm{G}}^{2} /\left(\sigma_{\mathrm{G}}^{2}+\sigma_{\text {error }}^{2} / \mathrm{r}\right)$, where $\sigma_{\mathrm{G}}^{2}$ denotes the genotypic variance, $\sigma_{\text {error }}^{2}$ the error variance, and $r$ the replicate number (Holland et al. 2003). Distributions of residuals were checked for normality using PROC UNIVARIATE (Shapiro-Wilk statistics). Correlation coefficients for AUDPC among the five experiments were estimated applying PROC CORR (Pearson).

For each of the two trials testing the combination between $Q Y r$. cau-6DL and $\operatorname{Yr} 18$ (represented by the DNA markers gpw5179 and cssfr5, respectively), an ANOVA was conducted using PROC GLIMMIX with the DNA marker class and replicate effects as fixed and the overall error term as random. Model fits were evaluated through the residual plots and fit statistics. The original disease data (i.e., severity or proportion of the surface area diseased) were $\log _{10^{-}}$ transformed to improve model fits. Comparisons of means among marker classes were performed according to least significance difference (LSD) at $\alpha=0.05$ applying an SAS macro (Piepho 2012).
Means of the marker classes were also calculated based on the original disease severity. An individual experimental unit in terms of statistical test is the average over the 160 flag leaves $\left(20 \mathrm{~F}_{2: 3}\right.$ families $\times$ 8 leaves/family) of each marker class within a replicate.

\section{Results}

Qing Shumai showed consistently low stripe rust severities (never exceeding 30\%) in the trials conducted from 1995 to 2016 at the adult plant growth stage (Table 1), even though it was susceptible at the seedling stage (Supplementary Fig. S1). In contrast, the severities of the susceptible control Mingxian 169 were higher than $80 \%$. These results agree with those of the trials performed from 1987 to 1994 as reported by Z. J. Zhang (1995), indicating that the resistance of Qing Shumai is considerably stable.

Detection and mapping of QTL. In the five field trials for the Mingxian $169 \times$ Qing Shumai mapping population, severe stripe rust was induced in all test locations and years except for 2011 when the disease occurred less severely. Mean severities were between $86 \%$ (2011) and 98\% (2009) on Mingxian 169, whereas lower than 25\% on Qing Shumai was consistently observed across all the five test environments. To improve QTL mapping, AUDPC was calculated for each of the 276 RILs. ANOVA revealed highly significant effects due to RIL on AUDPC $(P<0.0001)$ across all five environments and within each of them (Table 2 ). The variations across replicates within individual environments were relatively small $(P>0.01)$. The effect of environment was large (Table 2), indicating that substantial difference in conduciveness to stripe rust was established for testing the resistance. While the RIL-by-environment interaction variance was significant, high heritability (0.97) was observed across all five test environments (Table 2), suggesting the predominance of genotypic (i.e., RIL) variance over the interaction variance. Genotypic effect was also significant $(P<0.0001)$ when the RIL mean square was tested against the mean square of the RIL-by-environment interaction. Heritability was also high within each environment, implying that major QTL might be involved in the Mingxian $169 \times$ Qing Shumai population. As shown in Figure 1, AUDPC values of RILs varied widely from 124 to 1,277 . The distributions were continuous and monomodal, suggesting a quantitative inheritance. A correlation analysis was conducted among the test locations and years for disease data. The $P$-value

Table 1. Stripe rust disease severity of the Chinese wheat landrace Qing Shumai and the susceptible control wheat Mingxian 169 infected by the naturally occurring populations of Puccinia striiformis f.sp. tritici in the field plots of southern Gansu Province during the period from 1995 to 2016

\begin{tabular}{|c|c|c|c|c|}
\hline \multirow[b]{2}{*}{ Year } & \multicolumn{2}{|r|}{ Qing Shumai } & \multicolumn{2}{|c|}{ Mingxian 169} \\
\hline & Mean & Standard deviation & Mean & Standard deviation \\
\hline 1995 & 20 & 2.5 & 95 & 2.6 \\
\hline 1996 & 21 & 2.6 & 92 & 3.8 \\
\hline 1997 & 13 & 3.0 & 83 & 4.4 \\
\hline 1998 & 16 & 2.6 & 91 & 2.1 \\
\hline 1999 & 21 & 2.6 & 92 & 2.5 \\
\hline 2000 & 21 & 2.6 & 95 & 3.1 \\
\hline 2001 & 23 & 3.0 & 93 & 3.8 \\
\hline 2002 & 30 & 2.6 & 94 & 4.0 \\
\hline 2003 & 21 & 3.1 & 92 & 3.5 \\
\hline 2004 & 17 & 2.6 & 87 & 3.1 \\
\hline 2005 & 13 & 3.1 & 88 & 3.1 \\
\hline 2006 & 15 & 3.1 & 87 & 3.1 \\
\hline 2007 & 11 & 2.1 & 82 & 4.0 \\
\hline 2008 & 21 & 3.0 & 96 & 3.5 \\
\hline 2009 & 22 & 3.1 & 98 & 1.0 \\
\hline 2010 & 18 & 3.1 & 92 & 3.6 \\
\hline 2011 & 8 & 2.6 & 86 & 5.5 \\
\hline 2012 & 20 & 3.2 & 96 & 2.1 \\
\hline 2013 & 10 & 2.0 & 88 & 4.2 \\
\hline 2014 & 21 & 3.5 & 95 & 2.1 \\
\hline 2015 & 17 & 1.5 & 93 & 3.2 \\
\hline 2016 & 20 & 2.5 & 96 & 2.5 \\
\hline
\end{tabular}


of each correlation coefficient was less than 0.0001 (Table 3 ), indicating a high similarity in ranking of RILs across these environments.

Of the 2,018 SSR and EST primer pairs screened initially, 614 produced polymorphic bands between the parents, and 84 marker loci were clearly polymorphic between the R-and S-bulks. These markers were then used to genotype the 40 individual RILs constituting the contrasting bulks. For each marker, an ANOVA was conducted based on AUDPC values of the 40 RILs. The results showed that cfd 45 was the most significant marker $(F>95, P<0.0001)$ across all the three test seasons (see Materials and Methods section). According to the wheat consensus map from the website https://ccg.murdoch.edu.au/cmap/ccg-live/, cfd45 is located on the long arm of chromosome 6D (6DL). Thus, 6D was the focus for mapping, and a major QTL $(Q Y$ r.cau- $6 D L)$ linked with cfd45 was detected on 6DL (Fig. 2). To identify a marker linked with QYr.cau-6DL more tightly than cfd 45,326 SSR and EST primer pairs (in addition to the initial 2,018 ones) were screened, and gpw5179 was found to be tightly linked with QYr.cau-6DL (Fig. 2). The final length of the genetic map of 6D, constructed based on 276 RILs, was $129.1 \mathrm{cM}$ (Fig. 2). The resistance allele at QYr.cau- $6 D L$ was contributed by Qing Shumai. In all the trials, QYr.cau- $6 D L$ was significant with its peak LOD values varying from 5.9 (2011, southern Gansu) to 12.2 (2009, southern Gansu) (Fig. 2, Table 4). The effect size, as measured in $R^{2}$ (i.e., phenotypic variance explained by a QTL), ranged from $26.9 \%$ (2011) to $39.8 \%$ (2009) (Table 4).

Three additional QTL were detected on the chromosome arms 4AL, 4BL, and 5AS. Like QYr.cau-6DL, these three QTL were significant across all the test environments, with the resistance alleles being contributed by Qing Shumai (Supplementary Fig. S2). These QTLs showed smaller effect sizes $\left(R^{2}<16 \%\right)$ than did QYr.cau-6DL $\left(R^{2}\right.$ up to $39.8 \%$ ). The distance between the flanking markers was longer that $20 \mathrm{cM}$ for each of the three QTL, i.e., no tightly linked marker has yet been identified for these QTL, although 2,234 primer pairs were screened in total. Selection based on such a loosely linked marker may not be adequately reliable for practical breeding. Further efforts are being dedicated to the three QTL, while only $Q Y$ r.cau-6DL is addressed in this paper.
Effect of the combination of QYr.cau-6DL with Yr18. The four DNA marker classes $[(+,-),(-,+),(+,+)$, and $(-,-)]$ of RL6058 $\times$ Qing Shumai $\mathrm{F}_{2: 3}$ families were tested in a spring wheat genetic background in the 2014-15 season and in a winter wheat background in 2015-16. A continuously high inoculum pressure was created in both seasons, and fairly good infection homogeneity was observed by checking rust severity on the susceptible control plants that were arranged throughout each trial (see Materials and Methods section). Thus, there was little chance for any flag leaf to escape inoculation, and the resistance or susceptibility of each marker class could be

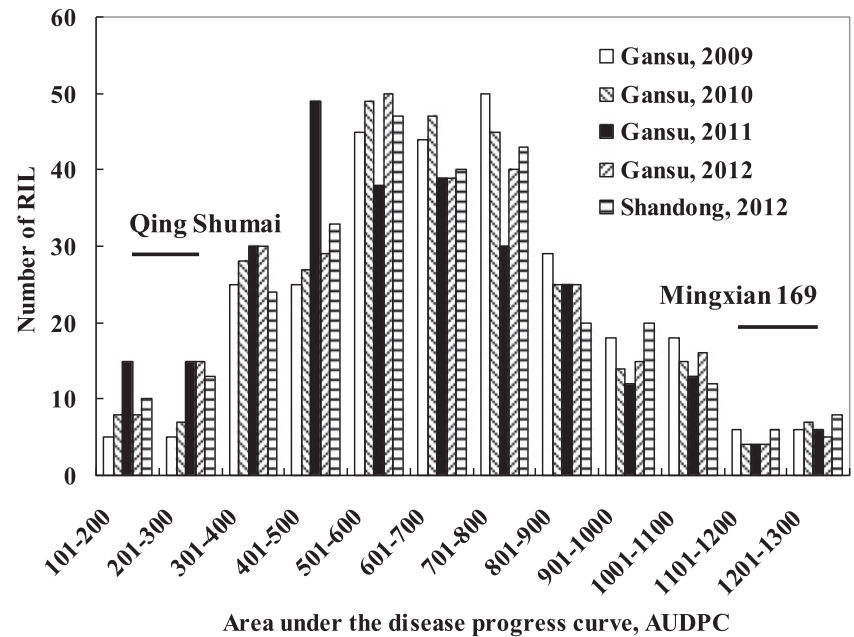

Fig. 1. Distribution of the 276 recombinant inbred lines (RILs) of the wheat cross Mingxian $169 \times$ Qing Shumai over the range of stripe rust infection as measured in AUDPC. The test plants were artificially inoculated with the Puccinia striiformis f. sp. tritici race CYR32 in the trial of Shandong Province, whereas infection occurred by the natural population of $P$. striiformis f. sp. tritici in southern Gansu Province. Horizontal bars indicate the approximate AUDPC ranges of the parental wheat lines.

Table 2. Analysis of variance (ANOVA) for disease intensity, as measured in the area under the disease progress curve, in the wheat cross Mingxian $169 \times$ Qing Shumai (276 recombinant inbred lines [RILs]) in five environments and across-environment analysis, and variance component analysis

\begin{tabular}{|c|c|c|c|c|c|c|}
\hline \multirow[b]{2}{*}{ Environment, Source } & \multicolumn{3}{|c|}{ ANOVA } & \multicolumn{2}{|c|}{ Variance component } & \multirow[b]{2}{*}{ Heritability } \\
\hline & $d f^{z}$ & $F$ value & $P$ value & Term & Values & \\
\hline \multicolumn{7}{|l|}{ Across environment } \\
\hline $\mathrm{RIL}(\mathrm{G})$ & 275 & 21.13 & $<0.0001$ & $\sigma_{\mathrm{G}}^{2}$ & $104,306.3$ & 0.97 \\
\hline Environment (E) & 4 & 912.87 & $<0.0001$ & $\sigma_{\mathrm{E}}^{2}$ & $4,446,307.7$ & \\
\hline $\mathrm{RIL} \times \mathrm{E}$ & 1097 & 1.96 & $<0.0001$ & $\sigma_{\mathrm{G} \times \mathrm{E}}^{2}$ & $9,535.1$ & \\
\hline Replicate $(\mathrm{R})$ within $\mathrm{E}$ & & & & $\sigma_{\mathrm{R} \text { within } \mathrm{E}}^{2}$ & $6,084.5$ & \\
\hline Error & & & & $\sigma_{\text {error }}^{2}$ & $4,870.7$ & \\
\hline \multicolumn{7}{|l|}{ Gansu, 2009} \\
\hline $\operatorname{RIL}(\mathrm{G})$ & 275 & 39.26 & $<0.0001$ & $\sigma_{\mathrm{G}}^{2}$ & $176,073.1$ & 0.98 \\
\hline Replicate (R) & 2 & 1.74 & 0.1761 & $\sigma_{\mathrm{R}}^{2}$ & $7,803.543$ & \\
\hline Error & & & & $\sigma_{\text {error }}^{2}$ & $4,623.5$ & \\
\hline \multicolumn{7}{|l|}{ Gansu, 2010} \\
\hline RIL (G) & 275 & 15.28 & $<0.0001$ & $\sigma_{\mathrm{G}}^{2}$ & $72,538.39$ & 0.97 \\
\hline Replicate (R) & 2 & 3.70 & 0.0254 & $\sigma_{\mathrm{R}}^{2}$ & $17,564.92$ & \\
\hline Error & & & & $\sigma_{\text {error }}^{2}$ & $4,894.1$ & \\
\hline \multicolumn{7}{|l|}{ Gansu, 2011} \\
\hline RIL (G) & 273 & 12.47 & $<0.0001$ & $\sigma_{\mathrm{G}}^{2}$ & $147,241.4$ & 0.97 \\
\hline Replicate (R) & 2 & 4.06 & 0.0178 & $\sigma_{\mathrm{R}}^{2}$ & $47,889.5$ & \\
\hline Error & & & & $\sigma_{\text {error }}^{2}$ & $12,210.6$ & \\
\hline \multicolumn{7}{|l|}{ Gansu, 2012} \\
\hline RIL (G) & 275 & 22.46 & $<0.0001$ & $\sigma_{\mathrm{G}}^{2}$ & $101,166.5$ & 0.98 \\
\hline Replicate (R) & 2 & 2.14 & 0.1191 & $\sigma_{\mathrm{R}}^{2}$ & $4,490.3$ & \\
\hline Error & & & & $\sigma_{\text {error }}^{2}$ & $4,653.2$ & \\
\hline \multicolumn{7}{|l|}{ Shandong, 2012} \\
\hline RIL $(G)$ & 274 & 19.37 & $<0.0001$ & $\sigma_{\mathrm{G}}^{2}$ & $88,940.6$ & 0.98 \\
\hline Replicate (R) & 2 & 2.38 & 0.0935 & $\sigma_{\mathrm{R}}^{2}$ & $10,950.6$ & \\
\hline Error & & & & $\sigma_{\text {error }}^{2}$ & $4,709.4$ & \\
\hline
\end{tabular}

${ }_{\mathrm{z}} \mathrm{df}=$ degree of freedom. 
revealed fully. ANOVA illustrated highly significant $(P<0.0001)$ effects due to DNA marker class on disease intensity and insignificant replicate effect (Table 5). Disease severity means were summarized in Table 6 for each marker class. Five points can be seen based on the comparisons among these values. First, the severities (27.5 to $30.6 \%)$ of the class $(-,+)$ were lower than those $(68.4$ to $74.6 \%)$ of $(-,-)$, indicating that the selection based on cssfr 5 for $Y r 18$ was effective. Second, severities (24.9 to $28.6 \%$ ) of (+, -) were lower than those (68.4 to $74.6 \%)$ of $(-,-)$, illustrating the effectiveness of selection based on gpw5179 for QYr.cau-6DL. Third, severities (14.5 to 15.8\%) of $(+,+)$ were lower than those $(24.9$ to $30.6 \%)$ of $(+,-)$ and $(-,+)$. This result shows that the simultaneous selection based on gpw5179 and cssfr5 yielded a larger effect in comparison with the separate selection based on either gpw5179 or cssfr 5 . Fourth, severities of $(+,-)$ were not statistically different $(\alpha=0.05)$ from those of $(-,+)$, although $(+,-)$ seems to be somewhat more resistant than $(-,+)$. This result supports the conclusion that the effect size of QYr.cau-6DL was approximately equal to that of Yr18. Last, all of these four results were consistent across both spring and winter wheat backgrounds. In addition, the standard deviation values of $(+,-)$ were smaller than those of $(-,+)$ in both trials. This constitutes a suggestion that $Q Y$ r.cau- $6 D L$ might be more stable than $\operatorname{Yr} 18$.

\section{Discussion}

In this study, we found a major QTL (QYr.cau-6DL) for stripe rustresistance effective at the adult plant growth stage from the Chinese wheat landrace Qing Shumai. QYr.cau-6DL was mapped on the long

Table 3. Pearson correlation coefficients $\mathrm{z}^{\mathrm{z}}$ among the disease data, recorded in five test environments, of the 276 recombinant inbred lines from the cross Mingxian $169 \times$ Qing Shumai

\begin{tabular}{lccccc}
\hline Test environment & $\begin{array}{c}\text { Gansu, } \\
\text { 2008-09 }\end{array}$ & $\begin{array}{c}\text { Gansu, } \\
\text { 2009-10 }\end{array}$ & $\begin{array}{c}\text { Ga10-11 } \\
\text { 2011-12 }\end{array}$ & $\begin{array}{c}\text { Shandong, } \\
\text { 2011-12 }\end{array}$ \\
\hline Gansu, 2008-09 & 1 & 0.88 & 0.78 & 0.91 & 0.86 \\
Gansu, 2009-10 & & 1 & 0.75 & 0.84 & 0.81 \\
Gansu, 2010-11 & & & 1 & 0.77 & 0.73 \\
Gansu, 2011-12 & & & & 1 & 0.82 \\
Shandong, 2011-12 & & & & & 1 \\
\hline
\end{tabular}

${ }^{\mathrm{z}}$ The $P$ value was less than 0.0001 for each of the correlation coefficients.

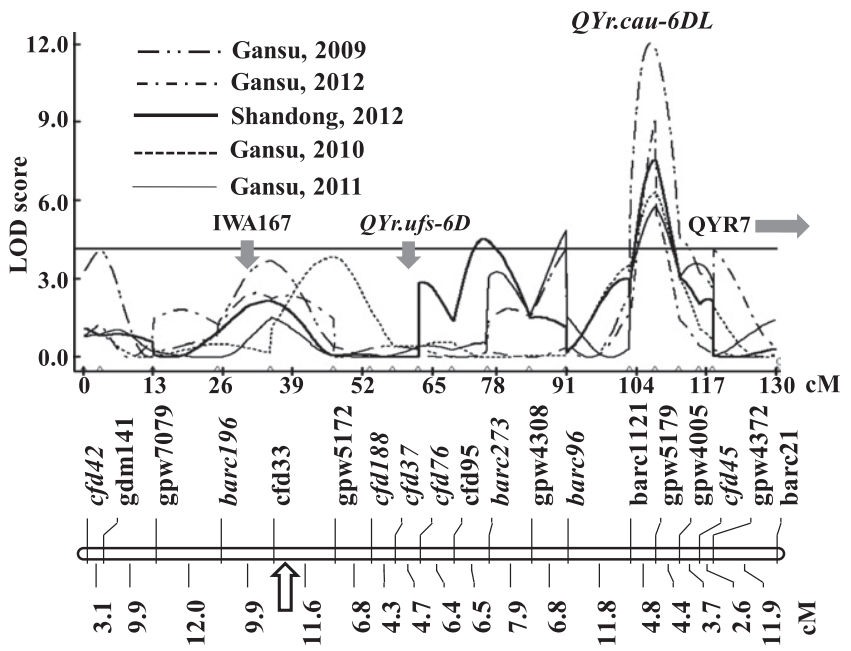

Fig. 2. Linkage group assigned to chromosome $6 D$ in the wheat cross Mingxian $169 \times$ Qing Shumai, illustrating the LOD values of QYr.cau-6DL. DNA markers and their positions are shown along chromosome $6 \mathrm{D}$ with the telomere of the short arm to the left. Distances are in Kosambi cM. The upward arrow suggests the position of the centromere estimated by referring to the SSR map of Somers et al. (2004). The italicized markers are common with either of two consensus maps (Somers et al. 2004; the map from the website https://ccg.murdoch.edu.au/cmap/ccg-live/). Solid arrows indicate the approximate positions of the previously reported QYr.ufs- $6 D$ (Agenbag et al. 2012), QYR7 (Boukhatem et al. 2002), and an undesignated QTL represented by the marker IWA167 (Maccaferri et al. 2015). The horizontal line denotes the threshold LOD of 4.2 . arm of chromosome 6D (6DL). Four $Y r$ genes and three stripe rustresistance QTL were identified previously on 6D. All of them could be distinguished from QYr.cau-6DL. The four genes $Y r 20, Y r 23$, YrTye, and $Y r T r l$ were assigned to 6D by monosomic analysis without discerning 6DS or 6DL (X. M. Chen et al. 1995a, b). They are expressed at the seedling growth stage and characterized by hypersensitive response; thus, they should be different from QYr.cau$6 D L$ that showed slow rusting at the adult plant stage. Maccaferri et al. (2015) mapped the DNA marker IWA167 to 6DS and found that an undesignated QTL was closely linked with this marker. IWA167 is separated from QYr.cau-6DL by at least $60 \mathrm{cM}$ (Fig. 2). The other two QTL (QYR7 and QYr.ufs-6D) were mapped to 6DL by Boukhatem et al. (2002) and Agenbag et al. (2012), respectively. QYR7 is flanked by the markers bed1510 and ksuD27, and QYr.ufs-6D sites in a region delimited by gwm325 and barc175. No common markers are available for the 6D map of the present study and those of Boukhatem et al. (2002) and Agenbag et al. (2012), hence, QYr.cau-6DL could not be spatially compared with QYR7 and QYr.ufs-6D directly. Nevertheless, indirect comparisons can be done by referring to certain wheat consensus maps. Eight markers (the italicized in Fig. 2) are common for the 6D map of the present study and the SSR consensus map of Somers et al. (2004) and/or the consensus map from the website https://ccg.murdoch.edu. $\mathrm{au} / \mathrm{cmap} / \mathrm{ccg}$-live/. With these maps as references, $Q Y$ r.cau- $6 D L$ was found to be proximal to QYR7 and distal to QYr.ufs-6D by more than $40 \mathrm{cM}$ and $30 \mathrm{cM}$, respectively (Fig. 2). Therefore, QYr.cau$6 D L$ is highly likely to be novel compared with the previously reported Yr genes and QTL.

Marker-based selection for QYr.cau- $6 D L$ and combination of QYr.cau-6DL with $Y r 18$. Yr18 has proven to be a very important durable resistance resource (Dyck et al. 1966; Krattinger et al. 2009; McIntosh 1992; Singh 1992). The DNA marker cssfr5 corresponds to a fragment of the cloned $\mathrm{Yr} 18$ gene sequence, being completely applicable to precise selection for $\operatorname{Yr} 18$ (Krattinger et al. 2009; Lagudah et al. 2009). Like other disease resistance QTL, Yr18 may not provide a commercially adequate level of resistance under high disease pressure when present alone (Suenaga et al. 2003). Additional QTL are needed to combine with $\operatorname{Yrl} 18$ for enhancing resistance. The RL6058 $\times$ Qing Shumai population was segregating for both Yrl8 and QYr.cau-6DL. Yrl8 and cssfr5 gave an excellent baseline for which $Q Y$ r.cau-6DL and gpw5179 could be compared. A simultaneous selection based on gpw5179 and cssfr5 yielded an elevated level of resistance in comparison with the separate selection based on either gpw5179 or cssfr5. These results indicate, on one hand, that

Table 4. The effect sizes of $Q Y$ r.cau-6DL, a quantitative trait locus (QTL) conferring stripe rust resistance, observed in five test environments

\begin{tabular}{lrc}
\hline Environment & LOD $^{\mathbf{z}}$ & $\boldsymbol{R}^{\mathbf{2}}$ \\
\hline Gansu, 2009 & 12.2 & 39.8 \\
Gansu, 2012 & 9.0 & 35.8 \\
Shandong, 2012 & 7.6 & 33.4 \\
Gansu, 2010 & 6.2 & 28.5 \\
Gansu, 2011 & 5.9 & 26.9 \\
\hline
\end{tabular}

${ }^{\mathrm{z}} \mathrm{LOD}=$ Logarithm of the odds.

Table 5. Analysis of variance based on the $\log _{10}$-transformed disease severities for the DNA marker classes of $\mathrm{F}_{2: 3}$ families, selected from the wheat cross Qing Shumai $\times$ RL6058, and the susceptible control wheat Mingxian 169

\begin{tabular}{lllrr}
\hline Genetic background & \multicolumn{1}{c}{ Source } & $\mathbf{d f}^{\mathbf{z}}$ & $\boldsymbol{F}$ value & $\boldsymbol{P}$ value \\
\hline Spring growth habit & & & & \\
& DNA marker class & 4 & 166.01 & $<0.0001$ \\
& Replicate & 2 & 0.33 & 0.7275 \\
Winter growth habit & & & & \\
& DNA marker class & 4 & 136.43 & $<0.0001$ \\
& Replicate & 2 & 0.05 & 0.9504 \\
\hline
\end{tabular}

${ }^{\mathrm{z}} \mathrm{df}=$ degree of freedom. 
the marker gpw5179 can be well applicable to the selection for QYr. cau-6DL if the marker is polymorphic in a given population and, on the other hand, that $Q Y$ r.cau- $6 D L$ and $Y r 18$ act well additively without undesired epistasis. A point to note is, as reviewed by Rosewarne et al. (2013), that $\operatorname{Yrl} 8$ generally acts additively with other stripe rustresistance QTL. However, Yr29 (another important resource similar to $\mathrm{Yr} 18$ ) may be less effective against stripe rust in the presence of Yr18, viz., they are not completely additive in the presence of each other. Rosewarne et al. (2013) assumed that $Y r 18$ and Yr29 might work in the same molecular pathway in inhibiting fungal growth. According to the present study, QYr.cau-6DL is superior to Yr29 with respect to combination with Yr18. QYr.cau-6DL may work in a distinct molecular pathway from that of $\operatorname{Yr} 18$, and this could contribute to the diversity of defense mechanisms.

Stable effectiveness of QYr.cau-6DL across different genetic backgrounds and environments. We believe that $Q Y$ r.cau- $6 D L$ possesses a good stability in effectiveness and thus has high potential value for breeding for the two following reasons. First, $Q Y$ r.cau- $6 D L$ is effective in both the crosses of Mingxian $169 \times$ Qing Shumai and RL6058 $\times$ Qing Shumai (Fig. 2, Table 6), and in the genetic backgrounds of both winter-type and spring-type wheat (Table 6). The Chinese winter wheat landrace Mingxian 169 is unlikely to share any common ancestor with the North American spring wheat Thatcher, i.e., these two crosses represent considerably diverse genetic backgrounds. Second, QYr.cau-6DL is effective in considerably diverse environments as constituted by the five field trials involving two test locations (southern Gansu and Shandong) and four years (Fig. 2). Agro-ecologically speaking, the two locations are representatives of the extreme wheat-growing regions in North and Northwest China (the main wheat production areas). The two locations are quite different in many aspects. For instance, they are geographically separated by a distance of approximately $1,100 \mathrm{~km}$. In southern Gansu, the trial plots were located at an elevation of 1,680 $\mathrm{m}$, and the autumn-sown wheat season was between mid-September and late July of the following year. The plots of Shandong were at an elevation of $90 \mathrm{~m}$ and the wheat season lasted from early October to the following June. In the wheat-growing months of spring and summer, the monthly mean temperatures ranged from 6.7 to $19.3^{\circ} \mathrm{C}$ in southern Gansu plots and from 10.3 to $28.2^{\circ} \mathrm{C}$ in Shandong plots (Du et al. 2015).

The effect size, as measured in $R^{2}$, of $Q Y$ r.cau- $6 D L$ varied from 26.9 to $39.8 \%$ (Table 4). Such a difference in effect magnitude might be attributed mainly to different degrees of infection homogeneity in the field trials. For instance, in southern Gansu, rainfalls in 2009 were quite frequent and the weather was favorable for P. striiformis to sporulate and reinfect. Abundant urediniospores were continuously released and dispersed over the whole trial. These provided a circumstance where any flag leaf might have little chance to escape inoculation. The high degree of infection homogeneity would reduce experimental error and thus lead to the large LOD and $\mathrm{R}^{2}$ values. In 2011, however, the weather was unusually dry, and sporulation and reinfection were constrained. Some flag leaves might not be adequately inoculated, and thus the effect of QYr.cau- $6 D L$ could not be revealed fully.
Evidence for durable stripe rust-resistance in relation to $Q Y r$. cau-6DL. We presume that $Q Y$ r.cau- $6 D L$ may be effective against a wide range of $P$. striiformis races and confer a type of durable resistance for the three following reasons. First, our field plots/nurseries were located in southern Gansu, a subarea of Northwest China that has been considered a "hotspot" of $P$. striiformis in the world by the rust pathologists in China (W. Q. Chen et al. 2009; Wan et al. 2004) and in other countries (Ali et al. 2010, 2014; Brown and Hovmøller 2002; Mboup et al. 2009; Wellings 2011). These pathologists report that $P$. striiformis isolates are extraordinarily diverse in southern Gansu where the wheat cropping systems, geomorphologic features, and weather conditions allow $P$. striiformis to readily survive year-round, and create a nursery or brooder for $P$. striiformis to raise new virulence races. Historically, almost all new Chinese $P$. striiformis races were first detected in this hotspot area (W. Q. Chen et al. 2009; Wan et al. 2004). We could identify as many as 18 races from only 62 P. striiformis samples collected from our plots of southern Gansu in 2012 (Z. J. Zhang, unpublished data). Second, we have observed Qing Shumai in our field nurseries in southern Gansu for many years. Stripe rust severity on Qing Shumai never exceed $25 \%$ in all 30 years from 1987 to 2016 except for 1990 and 2002, when approximately $30 \%$ severity was observed; this is relative to severity levels of $90 \%$ on the susceptible control Mingxian 169 (Table 1; Z. J. Zhang 1995). For the period from 2003 to 2007, $P$. striiformis race identification results are available as reported by W. Q. Chen et al. (2009), showing that 41 races were found. In the presence of these races, Qing Shumai consistently exhibited lower stripe rust intensities, implying the effectiveness of the resistance against the great number of races. QYr.cau-6DL, the largest QTL detected in Qing Shumai, should be the major contributor to this resistance. Last, as found in the files/records (accession numbers: F-7, F-26, F-230, and F-266) at the Scientech Documentation and Information Centre of Chinese Academy of Agricultural Sciences (Beijing) and the information from veteran farmers as detailed in Z. J. Zhang (1995), Qing Shumai was cultivated in southern Gansu from the year 1880 to 1968 . Approximately 38,000 hectares of wheat land were covered by Qing Shumai annually from the early 1940s to the early 1960s, illustrating that this landrace was widely grown. During the 1950 s and 1960s, stripe rust severities of approximately $25 \%$ were observed on Qing Shumai by plant pathologists, wheat breeders, and farmers as reported in Z. J. Zhang (1995). No disease assessment data are available for the period before 1950. However, it is reasonable to assume that Qing Shumai might not be heavily infected by $P$. striiformis; otherwise, farmers would not cultivate it on a large acreage for a long period of time in a $P$. striiformis hotspot. Johnson (1992) coins the term "durable resistance" describing "the resistance that remains effective in a cultivar that is widely grown for a long period of time in an environment favorable to the disease." This means that the diagnostic test for distinguishing between durable resistance and race-specific resistance is durability itself, and it can be confirmed only retrospectively over the course of time. This is a difficult but important task. The results of the present study combined with the historical files, records, and the gleaned pieces of information

Table 6. Stripe rust disease means of the four DNA marker classes of $\mathrm{F}_{2: 3}$ families selected from the wheat cross RL6058 $\times$ Qing Shumai for $Q Y$ r.cau- $6 D L$ and Yr18 based on the markers gpw5179 and cssfr5, respectively

\begin{tabular}{|c|c|c|c|c|c|c|}
\hline \multirow[b]{2}{*}{ DNA marker class $\mathbf{y}$} & \multicolumn{3}{|c|}{ Spring growth habit, 2014-15 season $^{z}$} & \multicolumn{3}{|c|}{ Winter growth habit, 2015-16 season ${ }^{z}$} \\
\hline & $\log _{10}$-mean & DS-mean & DS-standard deviation & $\log _{10}$-mean & DS-mean & DS-standard deviation \\
\hline$\overline{(-,+)}$ & $1.4366 \mathrm{~b}$ & 27.5 & 3.8 & $1.4829 \mathrm{~b}$ & 30.6 & 4.1 \\
\hline$(+,-)$ & $1.3963 \mathrm{~b}$ & 24.9 & 1.4 & $1.4554 \mathrm{~b}$ & 28.6 & 2.3 \\
\hline$(+,+)$ & $1.1951 \mathrm{a}$ & 15.8 & 2.1 & $1.1581 \mathrm{a}$ & 14.5 & 2.2 \\
\hline$(-,-)$ & $1.8346 \mathrm{c}$ & 68.4 & 3.0 & $1.8722 \mathrm{c}$ & 74.6 & 4.8 \\
\hline \multirow[t]{2}{*}{ S-Check } & $1.9675 \mathrm{~d}$ & 92.8 & 3.2 & $1.9835 \mathrm{~d}$ & 96.3 & 2.5 \\
\hline & $\mathrm{LSD}_{0.05}=0.0817$ & & & $\operatorname{LSD}_{0.05}=0.0937$ & & \\
\hline
\end{tabular}

$\mathrm{y}(-,+)=$ the susceptible $(\mathrm{S})$ allele in homozygous state at gpw5179 and resistant $(\mathrm{R})$ allele at $\operatorname{cssfr} 5,(+,-)=\mathrm{R}$-allele at gpw5179 and $\mathrm{S}$-allele at $\operatorname{cssfr} 5,(+,+)=$ R-allele at both gpw5179 and cssfr5, (-, -) = S-allele at both gpw5179 and cssfr5, and S-Check = the susceptible control wheat line Mingxian 169.

${ }^{\mathrm{z}}$ DS-mean denotes the means calculated based on the original disease severity values, while $\log _{10}$-mean denotes the means based on the $\log _{10}$-transformed disease severities. Values within the same column followed by different letters are significantly different according to least significant difference (LSD) test at $\alpha=0.05$. 
support the presumption that QYr.cau-6DL may confer a form of durable resistance sensu Johnson.

For application to practical breeding, further work is needed to clarify that $Q Y$ r.cau- $6 D L$ has no association with any undesired effect on wheat grain morphological and phenological traits. To increase diversity of defense mechanisms, further examinations should be dedicated to pyramiding QYr.cau-6DL, Yr18, and the high-temperature adult-plant resistance QTL such as Yr36 (Fu et al. 2009). QYr.cau$6 D L$ also needs to be further characterized with respect to resistance components.

\section{Acknowledgments}

This study was supported by the National Natural Science Foundation of China (30871612), the National Basic Research Program of China (2013CB127700), and the Chinese Universities Scientific Fund (2015NX005)

\section{Literature Cited}

Agenbag, G. M., Pretorius, Z. A., Boyd, L. A., Bender, C. M., and Prins, R. 2012. Identification of adult plant resistance to stripe rust in the cultivar CappelleDesprez. Theor. Appl. Genet. 125:109-120.

Ali, S., Gladieux, P., Leconte, M., Gautier, A., Justesen, A. F., Hovmøller, M. S., Enjalbert, J., and de Vallavieille-Pope, C. 2014. Origin, migration routes and worldwide population genetic structure of the wheat yellow rust pathogen Puccinia striiformis f.sp. tritici. PLoS Pathog. 10:e1003903.

Ali, S., Leconte, M., Walker, A. S., Enjalbert, J., and de Vallavieille-Pope, C. 2010. Reduction in the sex ability of worldwide clonal populations of Puccinia striiformis f. sp. tritici. Fungal Genet. Biol. 47:828-838.

Boukhatem, N., Baret, P. V., Mingeot, D., and Jacquemin, J. M. 2002. Quantitative trait loci for resistance against yellow rust in two wheat-derived recombinant inbred line populations. Theor. Appl. Genet. 104:111-118.

Brown, J. K. M., and Hovmøller, M. S. 2002. Aerial dispersal of pathogens on the global and continental scales and its impact on plant disease. Science 297: 537-541.

Chen, W. Q., Wu, L. R., Liu, T. G., Xu, S. C., Jin, S. L., Peng, Y. L., and Wang, B. T. 2009. Pathotype dynamics, diversity and virulence evolution in Puccinia striiformis f. sp. tritici, the causal agent of wheat stripe (yellow) rust in China from 2003 to 2007. Plant Dis. 93:1093-1101.

Chen, X. M., Jones, S. S., and Line, R. F. 1995a. Chromosomal location of genes for stripe rust resistance in spring wheat cultivars Compair, Fielder, Lee and Lemhi and interactions of aneuploid wheats with races of Puccinia striiformis. Phytopathology 85:375-381.

Chen, X. M., Line, R. F., and Jones, S. S. 1995b. Chromosomal location of genes for resistance to Puccinia striiformis in winter wheat cultivars Heines VII, Clement, Moro, Tyee, Tres and Daws. Phytopathology 85:1362-1367.

Dangl, J. L., Horvath, D. M., and Staskawicz, B. J. 2013. Pivoting the plant immune system from dissection to deployment. Science 341:746-751.

Dracatos, P. M., Zhang, P., Park, R. F., McIntosh, R. A., and Wellings, C. R. 2016. Complementary resistance genes in wheat selection 'Avocet $\mathrm{R}$ ' confer resistance to stripe rust. Theor. Appl. Genet. 129:65-76.

Du, Z. Y., Che, M. Z., Li, G. H., Chen, J., Quan, W., Guo, Y., Wang, Z., Ren, J. D., Zhang, H. Y., and Zhang, Z. J. 2015. A QTL with major effect on reducing leaf rust severity on the short arm of chromosome $1 \mathrm{~A}$ of wheat detected across different genetic backgrounds and diverse environments. Theor. Appl. Genet. 128:1579-1594.

Dyck, P. L., and Samborski, D. J. 1982. The inheritance of resistance to Puccinia recondita in group of common wheat cultivars. Can. J. Genet. Cytol. 24:273-283.

Dyck, P. L., Samborski, D. J., and Anderson, R. G. 1966. Inheritance of adult plant leaf rust resistance derived from the common wheat varieties Exchange and Frontana. Can. J. Genet. Cytol. 8:665-671.

Flor, H. H. 1971. Current status of the gene-for-gene concept. Annu. Rev. Phytopathol. 9:275-296.

Fu, D., Szucs, P., Yan, L., Helguera, M., Skinner, J. S., Von Zitzewitz, J., Hayes, P. M., and Dubcovsky, J. 2005. Large deletions within the first intron in VRN-1 are associated with spring growth habit in barley and wheat. Mol. Genet. Genomics 273:54-65.

Fu, D., Uauy, C., Distelfeld, A., Blechl, A., Epstein, L., Chen, X., Sela, H., Fahima, T., and Dubcovsky, J. 2009. A kinase-START gene confers temperature-dependent resistance to wheat stripe rust. Science 323:1357-1360.

Holland, J. B., Nyquist, W. E., and Cervantes-Martinez, C. 2003. Estimating and interpreting heritability for plant breeding: an update. Plant Breed. Rev. 22:9-112.

Hovmøller, M. S., Yahyaoui, A. H., Milus, E. A., and Justesen, A. F. 2008. Rapid global spread of two aggressive strains of a wheat rust fungus. Mol. Ecol. 17: 3818-3826.

Johnson, R. 1992. Reflections of a plant pathologist on breeding for disease resistance, with emphasis on yellow rust and eyespot of wheat. Plant Pathol. 41:239-254.

Krattinger, S. G., Lagudah, E. S., Spielmeyer, W., Singh, R. P., Huerta-Espino, J., McFadden, H., Bossolini, E., Selter, L. L., and Keller, B. 2009. A putative ABC transporter confers durable resistance to multiple fungal pathogens in wheat. Science 323:1360-1363.

Lagudah, E. S., Krattinger, S. G., Herrera-Foessel, S., Singh, R. P., Huerta-Espino, J., Spielmeyer, W., Brown-Guedira, G., Selter, L. L., and Keller, B. 2009. Genespecific markers for the wheat gene $L r 34 / Y r 18 / P m 38$ which confers resistance to multiple fungal pathogens. Theor. Appl. Genet. 119:889-898.

Lander, E. S., Green, P., Abrahamson, J., Barlow, A., Daly, M. J., Lincoln, S. E., and Newburg, L. 1987. MAPMAKER: an interactive computer package for constructing primary genetic linkage maps of experimental and natural populations. Genomics 1:174-181

Li, Z. F., Xia, X. C., Zhou, X. C., Niu, Y. C., He, Z. H., Zhang, Y., Li, G. Q., Wan, A. M., Wang, D. S., Chen, X. M., Lu, Q. L., and Singh, R. P. 2006. Seedling and slow rusting resistance to stripe rust in Chinese common wheats. Plant Dis. 90:1302-1312.

Maccaferri, M., Zhang, J., Bulli, P., Abate, Z., Shiaoman, C., Cantu, D., Bossolini, E., Chen, X., Pumphrey, M., and Dubcovsky, J. 2015. A genome-wide association study of resistance to stripe rust (Puccinia striiformis f. sp. tritici) in a worldwide collection of hexaploid spring wheat (Triticum aestivvum L.). Genes Genom. Genet. 5 (G3):449-465.

Mboup, M., Leconte, M., Gautier, A., Wan, A. M., Chen, W. Q., de VallaviellePope, C., and Enjalbert, J. 2009. Evidence of genetic recombination in whea yellow rust population of a Chinese over-summering area. Fungal Genet. Biol. 46:299-307.

McDonald, B. A., and Linde, C. 2002. Pathogen population genetics, evolutionary potential, and durable resistance. Annu. Rev. Phytopathol. 40:349-379.

McIntosh, R. A. 1992. Close genetic linkage of genes conferring adult-plant resistance to leaf rust and stripe rust in wheat. Plant Pathol. 41:523-527.

McIntosh, R. A., Dubcovsky, J., Rogers, W. J., Morris, C., Appels, R., and Xia, X. C. 2013a. Catalogue of gene symbols for wheat: 2013-2014 Supplement Online publication. https://wheat.pw.usda.gov/GG2/Triticum/wgc/2013/2013 2014_Supplement.pdf

McIntosh, R. A., Yamazaki, Y., Dubcovsky, J., Rogers, W. J., Morris, C., Appels, R., and Xia, X. C. 2013b. Catalogue of gene symbols for wheat in 12th International Wheat Genetics Symposium. R.A. McIntosh, ed. Yokohama, Japan.

Milus, E. A., and Line, R. F. 1986. Number of genes controlling hightemperature, adult-plant resistance to stripe rust in wheat. Phytopathology 76:93-96.

Peterson, R. F., Campbell, A. B., and Hannah, A. E. 1948. A diagrammatic scale for estimating rust intensity of leaves and stems of cereals. Can. J. Res. 26c: 496-500.

Piepho, H. 2012. A SAS macro for generating letter displays of pairwise mean comparisons. Commun. Biometry Crop Sci. 7:4-13.

Rosewarne, G. M., Herrera-Foessel, S. A., Singh, R. P., Huerta-Espino, J., Lan, C. X., and He, Z. H. 2013. Quantitative trait loci of stripe rust resistance in wheat. Theor. Appl. Genet. 126:2427-2449.

Singh, R. P. 1992. Genetic association of leaf rust resistance gene Lr34 with adult plant resistance to stripe rust in bread wheat. Phytopathology 82:835-838

Singh, R. P., Huerta-Espino, J., Bhavani, S., Herrera-Foessel, S. A., Singh, D. Singh, P. K., Velu, G., Mason, R. E., Jin, Y., Njau, P., and Crossa, J. 2011. Race non-specific resistance to rust diseases in CIMMYT spring wheats. Euphytica 179:175-186.

Singh, R. P., Huerta-Espino, J., and Rajaram, S. 2000. Achieving near immunity to leaf and stripe rusts in wheat by combining slow rusting resistance genes. Acta Phytopathol. Entomol. Hung. 35:133-139.

Somers, D. J., Isaac, P., and Edwards, K. 2004. A high-density microsatellite consensus map for bread wheat (Triticum aestivum L.). Theor. Appl. Genet. 109:1105-1114

Suenaga, K., Singh, R. P., Huerta-Espino, J., and William, H. M. 2003. Microsatellite markers for genes $\mathrm{Lr} 34 / \mathrm{Yr} 18$ and other quantitative trait loci for leaf rust and stripe rust resistance in bread wheat. Phytopathology 93:881-890.

Wan, A. M., Zhao, Z. H., Chen, X. M., He, Z. H., Jin, S. L., Jia, Q. Z., Yao, G., Yang, J., Wang, B., Li, G., Bi, Y., and Yuan, Z. 2004. Wheat stripe rust epidemic and virulence of Puccinia striiformis f. sp tritici in China in 2002 Plant Dis. 88:896-904.

Wang, S., Basten, J. C., and Zeng, Z. B. 2010. Windows QTL Cartographer 2.5 Department of Statistics, North Carolina State University, Raleigh, NC. http://statgen.ncsu.edu/qtlcart/WQTLCart.htm, Accessed 25 June 2015

Wellings, C. R. 2011. Global status of stripe rust: a review of historical and current threats. Euphytica 179:129-141.

William, H. M., Singh, R. P., Huerta-Espino, J., Palacios, G., and Suenaga, K. 2006. Characterization of genetic loci conferring adult plant resistance to leaf rust and stripe rust in spring wheat. Genome 49:977-990.

Yan, L., Helguera, M., Kato, K., Fukuyama, S., Sherman, J., and Dubcovsky, J. 2004. Allelic variation at the $V R N-1$ promoter region in polyploid wheat. Theor. Appl. Genet. 109:1677-1686

Zhang, X., Xiao, Y., Zhang, Y., Xia, X., Dubcovsky, J., and He, Z. 2008. Allelic variation at the vernalization genes Vrn-A1,Vrn-B1, Vrn-D1, and Vrn-B3 in Chinese wheat cultivars and their association with growth habit. Crop Sci. 48 : 458-470.

Zhang, Z. J. 1995. Evidence of durable resistance in nine Chinese landraces and one Italian cultivar of Triticum aestivum to Puccinia striiformis. Eur. J. Plant Pathol. 101:405-409. 